\title{
LA «DESINDUSTRIALIZACIÓN» EN COLOMBIA. UNA VERDAD A MEDIAS QUE OCULTA LA INEQUITATIVA DISTRIBUCIÓN DEL VALOR AGREGADO
}

THE "DEINDUSTRIALIZATION" IN COLOMBIA. A HALF-TRUTH THAT HIDE THE DISTRIBUTION INEQUITABLE OF ADDED VALUE.

“DESINDUSTRIALIZAÇÃO” NA COLÔMBIA. UMA MEIA VERDADE QUE ESCONDE A DISTRIBUIÇÃO DESIGUAL DO VALOR AGREGADO

SILVA-COLMENARES_Julio

PhD en Economía (summa cum laude) de la Escuela Superior de Economía de Berlín. Director del Observatorio sobre Desarrollo Humano, Universidad Autónoma de Colombia. Email: obdehumano@fuac.edu.co, Colombia.

Recibido: 24 de abril de 2018

Aprobación definitiva: Junio 12 de 2018

DOI: http://dx.doi.org/10.22267/rtend.181901.86

\section{RESUMEN}

Este artículo tiene como hipótesis que la «desindustrialización» en Colombia es una verdad a medias, sin que ello niegue la precaria situación de la industria. Para comprobar de manera empírica esta hipótesis se analiza la producción de los principales renglones y se compara su comportamiento en unidades físicas y en valores monetarios constantes, como una representación de los valores de uso y valores de cambio en la industria. Se comprueba que en 19 de los 28 renglones analizados, la producción en unidades físicas (valores de uso) aumentó a una tasa anual geométrica superior al incremento de la producción bruta en términos monetarios (valores de cambio, en precios constantes). Así pues, aunque la participación de 
la industria en el total del PIB se ha reducido, esto no se debe tanto a un proceso de desindustrialización, en sí, como a un mayor ritmo en el incremento de otras actividades económicas y a una reducción de costos unitarios por aumentos en la productividad bruta, en especial por cambios tecnológicos en los procesos, ya sea en la utilización del trabajo humano o en los insumos incorporados a la producción.

Palabras clave: Industrialización, desindustrialización, producción industrial, valores de uso y valores de cambio.

JEL: E23, L60, O14.

\section{ABSTRACT}

This article has the hypothesis that the "deindustrialization" in Colombia is a half-truth, without denying the precarious situation of the industry. To test empirically this hypothesis, the production of the main lines is analyzed and its behavior is compared in physical units and in constant monetary values, as a representation of the values of use and values of change in the industry. It is found that in 19 of the 28 lines analyzed, production in physical units (use values) increased at a geometric annual rate higher than the increase in gross production in monetary terms (exchange values, in constant prices). Thus, although the industry's participation in total GDP has been reduced, this is not so much due to a process of deindustrialization, in itself, as to a greater pace in the increase of other economic activities and a reduction in unit costs by increases in gross productivity, especially due to technological changes in processes, whether in the use of human labor or in the inputs incorporated into production.

Key words: Industrialization, deindustrialization, industrial production, use values and exchange values

JEL: E23, L60, O14. 


\section{RESUMO}

Este artigo tem a hipótese de que a "desindustrialização" na Colômbia é uma meia verdade, sem negar a situação precária da indústria. Para testar empiricamente esta hipótese, a produção das linhas principais é analisada e seu comportamento é comparado em unidades físicas e em valores monetários constantes, como uma representação dos valores de uso e valores de mudança na indústria. Verifica-se que em 19 das 28 linhas analisadas, a produção em unidades físicas (valores de uso) aumentou a uma taxa anual geométrica superior ao aumento da produção bruta em termos monetários (valores de troca, a preços constantes). Assim, embora a participação da indústria no PIB tenha sido reduzida, isso não se deve tanto a um processo de desindustrialização, por si só, quanto a um maior ritmo no aumento de outras atividades econômicas e à redução de custos unitários. por aumentos na produtividade bruta, especialmente devido a mudanças tecnológicas nos processos, seja no uso de mão-de-obra humana ou nos insumos incorporados à produção.

Palavras-chave: Industrialização, desindustrialização, produção industrial, valores de uso e valores de troca.

JEL: E23, L60, O14.

\section{INTRODUCCIÓN}

El presente artículo pretende comprobar de manera empírica que no existe un proceso de desindustrialización en Colombia. Esto no niega una situación actual precaria para la producción manufacturera, pero tampoco significa que la misma esté desapareciendo. La teoría objetiva del valor es el cristal a través del cual se revisa el comportamiento de la industria a través de las unidades físicas y monetarias reportadas en la Encuesta Anual Manufacturera que elabora el DANE, para los años 1994 y 2014, como una manera de determinar los cambios en términos de valores de uso y valores de cambio en los renglones más representativos, de acuerdo a la presencia de las empresas nacionales de mayor envergadura en el país. 


\section{MARCO TEÓRICO}

La inequitativa distribución del valor agregado se basa en la teoría objetiva del valor cuyo principal exponente es Carlos Marx. En ella, el trabajo humano no es un factor más de la producción, como lo dicen algunas corrientes del pensamiento económico, igualándolo a otros que entran en el proceso de producción, como la tierra, las máquinas o las materias primas, sino es el único, el factor por excelencia, capaz de transformar, de manera creativa, anteriores valores de uso en nuevos valores de uso, acrecentando la riqueza de la sociedad. Los demás elementos que entran en el proceso de trabajo sólo coadyuvan a darle forma a esa capacidad creadora, que depende, en lo fundamental, de la acumulación del conocimiento durante toda la historia de la humanidad. Como destaca el autor de estas líneas en otro artículo sobre el carácter dual del trabajo humano:

Marx ya no sólo habla de las dos caras de los bienes o mercancías, sino del carácter dual del trabajo humano, que constituye lo mejor de su libro, como él mismo se lo manifestó a Federico Engels (1820-1895) en carta de agosto de 1867, apenas concluida la preparación del primer tomo de El Capital'."Veíamos al comenzar -son sus palabras-- que la mercancía tenía dos caras: la del valor de uso y la del valor de cambio. Más tarde, hemos vuelto a encontrarnos con que el trabajo expresado en el valor no presentaba los mismos caracteres que el trabajo creador de valores de uso. Nadie, hasta ahora, había puesto de relieve críticamente este doble carácter del trabajo representado por la mercancía", y como prueba de este antecedente primigenio cita a pie de página su propio trabajo Contribución a la crítica de la economía. Y a continuación remata diciendo que "este punto es el eje en torno al cual gira la comprensión de la economía política" (Marx, 1959, págs. 8-9) citado en (Silva-Colmenares, 2014, pág. 41)

\footnotetext{
1 Carta de Marx a Engels: “...Lo que hay de mejor en mi libro es: 1. (y es sobre ello que descansa TODA la lucidez de los facts [hechos]) poner de relieve, desde el PRIMER capítulo, EL DOBLE CARÁCTER DEL TRABAJO, según se expresa en valor de uso o en valor de cambio; 2. el análisis de la PLUSVALÍA, INDEPENDIENTEMENTE DE SUS FORMAS PARTICULARES: ganancia, interés, renta del suelo, etc. El análisis de estas formas particulares en la economía clásica, que las confunde constantemente con la forma general, es una olla podrida". Carlos Marx, Federico Engels. Cartas sobre El Capital. Editorial de Ciencias Sociales, La Habana, 1976, pp. 176-177. (En la carta de Marx la expresión olla podrida está en español, por referencia a un plato típico de la gastronomía española, que mezcla varios tipos de carnes).
} 
Más adelante, se precisa el aporte de Marx a la distinción entre valores de uso y valores de cambio y a la singularidad del trabajo humano como el único factor de producción por su capacidad creadora de valores.

Marx distingue entre valor o valor de cambio, como encarnación de trabajo humano abstracto, o forma social de la riqueza, y valor de uso, como capacidad de un bien para satisfacer una necesidad, lo que de alguna manera se expresa siempre en trabajo humano concreto, o contenido material de la riqueza. Para reiterar que el trabajo ha sido desde siempre fuente de la riqueza, entendida como lo necesario para vivir, según la sabia expresión de Adam Smith, unas líneas más adelante Marx enfatiza: "Como creador de valores de uso, es decir como trabajo útil, el trabajo es, por tanto, condición de vida del hombre, y condición independiente de todas las formas de sociedad, una necesidad perenne y natural sin la que no se concebiría el intercambio orgánico entre el hombre y la naturaleza ni, por consiguiente, la vida humana" (Marx, 1959, pág. 10) citado en (Silva-Colmenares, 2014, pág. 42)²

\section{1 ¿Son los cambios relativos en el producto interno bruto y la ocupación en la industria sinónimo de «desindustrialización»?.}

Desde hace varios años el autor de estas páginas cree que la categoría «desindustrialización» no es la más apropiada para describir lo que ha ocurrido en la «industria manufacturera» en las últimas décadas -que cada vez es menos manufactura, o sea elaboración con las manos de los trabajadores--, para pasar a ser un proceso automatizado y, en algunos renglones, robotizado, con cambios sustanciales no sólo en los procesos de producción sino en las materias primas e insumos utilizados. No parece lógico hablar de «desindustrialización» cuando todos los días aumentan los productos de origen industrial -para lo cual no importa su procedencia, pues ocurre en casi todos los países-- que se utilizan tanto en los hogares como en todas las actividades económicas, incluidos, por ejemplo, servicios como la educación y la salud, cuya eficacia y eficiencia depende mucho de esta acelerada incorporación. Transformación que ha ocurrido con mayor facilidad en los países ricos, pues disponen de los recursos para hacerlo, pero también, quizá con menor intensidad y profundidad, en los países pobres, como es el caso de Colombia.

2 Para ver de forma más detallada los planteamientos teóricos que soportan este trabajo puede consultarse: (Silva-Colmenares, 2015) 
La mayoría de quienes plantean la tesis de la «desindustrialización» lo hacen, en lo fundamental, con base en la pérdida de peso relativo de la industria en el Producto Interno Bruto -PIB- y de la población ocupada -PO- en la industria, respecto a la PO total. Si se comparan estas cifras relativas en un lapso más o menos extenso, es evidente esa pérdida de peso. Por ejemplo, en la tabla 1 se muestra esta situación en el PIB de Colombia para los 20 años transcurridos entre 1994 y 2014, cuando el peso relativo de la industria disminuyó del $13,6 \%$ al $11.1 \%$; si se mira hacia décadas anteriores, en los años setenta y ochenta del siglo pasado había alcanzado a llegar a más del 23\% (Silva-Colmenares, Globalización, crecimiento y desarrollo,, 2013, pág. 149). Pero esta pérdida de peso relativo se explica por el hecho de que mientras la industria creció en estos 20 años a una tasa anual geométrica -TAGdel 2,3\%, y el conjunto de las actividades secundarias al 2,5\% anual, las actividades primarias aumentaron al 3,3\% anual y, sobre todo, las actividades terciarias crecieron al $3,7 \%$, como ha ocurrido en casi todos los países y lo muestra la tabla 1 para Colombia. Pero si la industria perdió peso relativo, su aporte en términos monetarios aumentó casi en $60 \%$, pues el PIB pasó de 36,4 billones en 1994 a 57,2 billones en 1994, en valores constantes de 2005.

\section{Tabla 1}

Composición del Producto Interno Bruto -PIBMiles de millones de pesos 2005

\begin{tabular}{|l|r|r|r|r|r|}
\hline \multirow{2}{*}{ Conceptos/años } & \multicolumn{2}{|c|}{ Producto interno bruto PIB } & \multicolumn{1}{c|}{ Composición \% del PIB } & TAG \\
\cline { 2 - 5 } & \multicolumn{1}{c|}{$\mathbf{1 9 9 4}$} & $\mathbf{2 0 1 4}$ & $\mathbf{1 9 9 4}$ & $\mathbf{2 0 1 4}$ & $\mathbf{1 9 9 5 - 2 0 1 4}$ \\
\hline Actividades primarias a/ & 36.341 & 69.465 & $13,6 \%$ & $13,4 \%$ & $3,29 \%$ \\
\hline Actividades secundarias a/ & 68.292 & 112.086 & $25,6 \%$ & $21,7 \%$ & $2,51 \%$ \\
\hline -- Industria manufacturera & 36.372 & 57.181 & $13,6 \%$ & $11,1 \%$ & $2,29 \%$ \\
\hline Actividades terciarias a/ & 135.520 & 280.467 & $50,8 \%$ & $54,3 \%$ & $3,70 \%$ \\
\hline Impuestos menos subvenciones & 26.609 & 54.601 & $10,0 \%$ & $10,6 \%$ & $3,66 \%$ \\
\hline Producto Interno bruto & 266.761 & 516.619 & $100,0 \%$ & $100,0 \%$ & $3,36 \%$ \\
\hline
\end{tabular}

Fuente: DANE - Dirección de Síntesis y Cuentas Nacionales

TAG - Tasa anual geométrica para el periodo indicado

a/ En actividades primarias se incluye agricultura, ganaderia, caza, pesca y silvicultura y la explotación de minas y canteras; en las actividades secundarias se incluyen la industria manufacturera, el suministro de electricidad, gas y agua y la construcción; en las actividades terciarias el resto de sectores. 
En el caso del empleo también se observa una situación similar, como puede apreciarse en la tabla 2. Mientras en el lapso 1991-2014, 23 años, la ocupación en la industria disminuyó del $15,0 \%$ al $11,6 \%$, esa ocupación en términos absolutos aumentó de 2,1 millones de personas a 2,5 millones, aunque con una tasa acumulativa baja, $0,78 \%$ anual, un poco menos de la mitad del ritmo al que creció la ocupación total, $1,9 \%$ anual, si bien ésta creció a un ritmo superior al crecimiento de la población total, que lo hizo a una TAG del 1,3\% entre 1994-2014, al pasar de 36,8 millones a 47,7 millones $^{3}$.

\section{Tabla 2}

Población ocupada - total nación e industria

Miles de personas

\begin{tabular}{|l|r|r|r|r|}
\hline Conceptos/años & \multicolumn{1}{|c|}{$\mathbf{1 9 9 1} \mathbf{a} /$} & \multicolumn{1}{c|}{$\mathbf{2 0 0 0} \mathbf{a} /$} & \multicolumn{1}{|c|}{$\mathbf{2 0 1 4} \mathbf{b} /$} & \multicolumn{1}{c|}{$\begin{array}{c}\text { TAG 1992- } \\
\mathbf{2 0 1 4}\end{array}$} \\
\hline Población ocupada Total & $14.027,7$ & $16.308,3$ & $21.614,9$ & $1,90 \%$ \\
\hline Población ocupada Industria & $2.101,2$ & $2.198,1$ & $2.510,9$ & $0,78 \%$ \\
\hline$\%$ Industria/Total & $15,0 \%$ & $13,5 \%$ & $11,6 \%$ & \\
\hline
\end{tabular}

TAG = Tasa anual geométrica para el periodo indicado

a/ Datos al final del año, tomados de Ramírez Manuel, Guevara Diego y Korena Ana, Mercado de trabajo y condiciones de empleo en Colombia: Los efectos de la globalización , 2003 (Trabajo de investigación realizadp para la Universidad Autónoma de Colombia. Fuente primaria: DANE)

b/ DANE, ECH - GEIH - Promedio trimestre julio-Septiembre

Sobre la base de las cifras relativas, un documento de una institución tan conocida como la Asociación Nacional de Instituciones Financieras -ANIF- dijo en el año 2013 que "Colombia ha venido experimentando un proceso de desindustrialización a través del cual su relación Valor Agregado Industrial/PIB ha venido descendiendo de niveles del $24 \%$ hace tres décadas a uno del $15 \%$ hace una década y actualmente se perfila hacia tan sólo 9\%-12\% en dicha relación hacia el período 20122020. En términos de generación de empleo, la industria aportaba cerca del $25 \%$ del total de empleo hace tres décadas, hace una década aportaba el $23 \%$, pero actualmente sólo contribuye con el $13 \% "$. Idea que los autores reiteran en las conclusiones (Clavijo, Vera, \& Fandiño, 2012, pág. 11 y 77 ).

3 Datos de DANE, Población Colombiana, tomados de www.dane.gov.co el 19 de febrero de 2018. 
Si se utilizan sólo las cifras porcentuales podría hablarse de una disminución relativa de la industria, pero si se toman las cifras absolutas mencionadas en párrafo anterior, sería discutible afirmar que «Colombia ha venido experimentando un proceso de desindustrialización». Por eso se habla de una verdad a medias, pues las cifras absolutas muestran otro panorama, lo que se confirma cuando se observa la producción industrial en unidades físicas, como se verá más adelante. No obstante, es indispensable estudiar el estancamiento relativo de la industria, esto es, en cuáles ramas es más crítico y cuáles pueden ser las razones explicativas de este cambio, sobre todo cuando está en curso la cuarta revolución industrial, que ha modificado de manera sustancial la forma cómo se hacen las cosas. Hay que encontrar la otra mitad de la verdad, pues la industria no ha desaparecido ni está en proceso de desaparecer, ya que lo que ocurre es un cambio de contenido o incluso de esencia, dadas las transformaciones sustanciales por las que pasa. Estos cambios la han llevado a perder peso relativo en el agregado nacional, sin que haya perdido importancia, como lo comprueba la discusión sobre la revolución industrial en marcha.

Incluso mejorías significativas en la actividad industrial, como por ejemplo la elevación de la productividad bruta (más unidades de producto por unidad de tiempo) o innovaciones en proceso y materiales (como la sustitución de minerales tradicionales pesados y caros por nuevas aleaciones livianas y más baratas), pueden llevar a reducciones importantes en costos unitarios, lo que hace que disminuya el monto dinerario y aumente el volumen producido, afectándose su peso relativo en el agregado nacional, medido en términos monetarios. Hoy se habla de una nueva producción que supone la sustitución de la fábrica de antaño, movida por el motor de vapor y la electricidad, con mucho trabajo humano "presencial y sencillo», por la empresa de hogaño, con bastante ciencia acumulada, que se mueve por impulsos electrónicos, y que utiliza poco trabajo humano «vivo» pero de alta especialización. El gran reto de hoy es buscarles empleo a los trabajadores que ya no pueden ocuparse en las labores «desaparecidas», lo que no es propósito de estas notas. Esto no niega que en el caso de Colombia haya que tener en cuenta aspectos colaterales que pueden haber disminuido la producción industrial, como el hecho de que muchas empresas industriales se han 
convertido en importadoras de bienes terminados -como ha ocurrido, por ejemplo, en las ramas de confecciones y productos químicos o en la industria automotriz y de máquinas-herramientas- o que muchas que producían en Colombia para exportar, ahora son productoras en el exterior, en un creciente proceso de «transnacionalización» del gran capital colombiano, lo que supone el traslado de sus procesos industriales a otros países ${ }^{4}$. Habría que estudiar con más detalle fenómenos propios de «deformación» en la estructura industrial en capitalismos "tardíos» y de «monopolización precoz», como el colombiano.

\subsection{Una hipótesis para la investigación}

Sin tener en cuenta la «transnacionalización» mencionada, de todas maneras puede plantearse la hipótesis de que hay una divergencia esencial en la industria entre la producción en unidades físicas y su expresión en valores monetarios constantes 5 . En categorías de la Economía Política habría que decir que mientras aumenta la producción en valores de uso (unidades físicas) disminuye, en términos relativos, la producción en valores de cambio (unidades monetarias constantes), pues se reduce, en su más amplia acepción, la utilización de trabajo humano en el proceso de producción. La hipótesis se encamina a demostrar que mientras la producción industrial en unidades físicas, esto es, en valores de uso, aumenta con el paso del tiempo, sobre todo por el crecimiento de la población y la diversificación y ampliación en la demanda física de los hogares, principal componente de la demanda total, la producción en valores constantes, lo que se entiende como valores de cambio en el mercado --pero hay que tener en cuenta que los valores utilizados en este trabajo corresponden a precios finales en fábrica, y no al consumidor final--, más bien disminuye por aumento de la productividad bruta, en especial por cambios tecnológicos en los procesos de trabajo, o modificaciones en las materias primas o los insumos utilizados en la producción o por cambios en la fuerza de trabajo, entre los principales; para precisar mejor este efecto se deben calcular y comparar los costos por unidad de medida, los que deben disminuir con el trascurso del tiempo.

4 Véase este proceso en: (Silva-Colmenares \& Padilla, 2015)

5 Una idea similar se desarrolla en el trabajo de (Silva-Colmenares, 2015) 
Así mismo influye en esta disminución el traslado que se ha hecho de actividades que antes se hacían por personal propio de las empresas industriales hacia otras empresas especializadas, las que tienen su propio personal, con mayor experiencia y productividad, aunque este efecto es menor cuando se trabaja sobre el valor de la producción bruta, pues parte de estos trabajos relocalizados la afectan como consumo interno. En ese sentido, hay que tener en cuenta que el concepto de producción bruta corresponde a la suma del valor agregado más el consumo intermedio --en donde se incluye esta compra de bienes o servicios a empresas que no son de la industria manufacturera, en sí--, por lo que no es comparable con el Producto Interno Bruto -PIB- de la industria, que en lo fundamental es valor agregado, aunque si puede influir en el cálculo de éste. Entre paréntesis puede decirse que muchas de esas empresas nuevas son filiales o subordinadas, con personería jurídica independiente, de la empresa matriz industrial o del conglomerado al que pertenece la matriz industrial.

\section{RESULTADOS.}

\subsection{Renglones industriales seleccionados y escogidos: cambios entre 1994 y 2014.}

El trabajo de grado que adelanta un estudiante de la Facultad de Ciencias Económicas, Administrativas y Contables de la Universidad Autónoma de Colombia sobre esta temática, hace algunos aportes a la hipótesis planteada en párrafo anterior (Puerto, En proceso de elaboración). Para este trabajo se seleccionaron, teniendo como criterio básico, pero no exclusivo, los renglones industriales con producción superior a 200 mil millones en el año 2014 en \$ de 2005 y cuya homogeneidad, sobre todo en unidades de medida, se haya mantenido sin modificación durante todo el lapso 1994-2014, y de ese grupo se escogieron algunos renglones que corresponden a consumos característicos de la llamada «canasta familiar» o típicos de la producción industrial. En total, fueron seleccionados 81 renglones industriales, de los cuales se escogieron 28 , casi una tercera parte, para un análisis más detallado. Para la expresión en valores constantes se deflactaron los valores corrientes reportados por la Encuesta Anual Manufacturera producida por el Departamento Nacional 
de Estadística -DANE- con el deflactor implícito del PIB, teniendo como año base el 2005; si bien hubiese sido más aconsejado hacerlo con un deflactor propio de la industria, el escogido tiene la ventaja que recoge el comportamiento de todos los precios de la economía colombiana, con la cual está en relación permanente la industria, lo que, como es comprensible, no garantiza exactitud, pero si permite comparabilidad, pues en este lapso no hubo grandes variaciones de precios.

\section{Tabla 3}

Producción bruta industria manufacturera y porcentaje que corresponde a renglones seleccionados y escogidos.

Valores en miles de millones de 2005

\begin{tabular}{|l|r|r|r|}
\hline \multicolumn{1}{|c|}{ Conceptos/años } & 1.994 & $\mathbf{2 . 0 1 4}$ & $\begin{array}{c}\text { TAG } \\
\text { Producción } \\
\text { total } \\
\mathbf{1 9 9 4 - 2 0 1 4}\end{array}$ \\
\hline Total producción industria manufacturera & 88.035 & 141.951 & $2,42 \%$ \\
\hline $\begin{array}{l}\text { \% Producción renglones seleccionados/Total producción industria } \\
\text { manufacturera }\end{array}$ & $45,2 \%$ & $45,2 \%$ & \\
\cline { 1 - 3 } $\begin{array}{l}\text { \% Producción renglones escogidos/ Total } \\
\text { producción industria manufacturera }\end{array}$ & $23,7 \%$ & $22,6 \%$ & \\
\hline
\end{tabular}

TAG: Tasa anual geométrica para el periodo indicado

Mientras la tabla 1 muestra que el PIB de la industria manufacturera aumentó a un ritmo del 2,29\% en tasa anual geométrica en el lapso 19942014 , la tabla 3 muestra que la producción bruta industrial aumentó a un ritmo un poco mayor, a una TAG del $2,42 \%$ anual, lo que significa que la industria incorpora cada vez más, aunque no en gran proporción, una mayor parte de consumo intermedio en el producto final. Otra forma de evidenciar esta incorporación es estableciendo la relación entre el PIB, que en la práctica es valor agregado, y la producción bruta industrial, que es la sumatoria de valor agregado y consumo intermedio. Para el año de 1994 el PIB equivalía al 41,3\% de la producción bruta, proporción que disminuyó al 40,3\% en 2014. En forma correlativa, el consumo intermedio aumentó del $58,7 \%$ al $59,7 \%$ en los mismos años, lo que significa que en la producción industrial más de la mitad del valor bruto final corresponde a bienes y servicios comprados a otras actividades económicas, con tendencia a aumentar. 


\section{Tabla 4}

Participación de los renglones seleccionados y escogidos en la producción total de la industria manufacturera 1994 y 2014 a/

Valores de miles de millones 2005

\begin{tabular}{|c|c|c|c|c|c|c|c|c|}
\hline \multirow[t]{2}{*}{ Rama industrial } & \multirow{2}{*}{$\begin{array}{c}\text { No. renglo- } \\
\text { nes selec- } \\
\text { cionados }\end{array}$} & \multirow{2}{*}{\begin{tabular}{|c|} 
No. \\
Renglo- \\
nes \\
escogi- \\
dos \\
\end{tabular}} & \multicolumn{2}{|c|}{$\begin{array}{c}\text { Valor producción } \\
\text { renglones } \\
\text { seleccionados }\end{array}$} & \multicolumn{2}{|c|}{$\begin{array}{l}\text { Valor producción } \\
\text { renglones escogidos }\end{array}$} & \multirow{2}{*}{\begin{tabular}{|} 
TAG 1995- \\
2014 Vlr. \\
reng. \\
selec.
\end{tabular}} & \multirow{2}{*}{$\begin{array}{l}\text { TAG 1995- } \\
2014 \text { Vlr. } \\
\text { reng. } \\
\text { escog. }\end{array}$} \\
\hline & & & 1994 & 2014 & 1994 & 2014 & & \\
\hline Alimentos y bebidas & 32 & 11 & 20.905 & 29.006 & 10.285 & 15.922 & $1,65 \%$ & $2,21 \%$ \\
\hline Textiles y confecciones & 10 & 3 & 1.952 & 3.596 & 624 & 1.188 & $3,10 \%$ & $3,27 \%$ \\
\hline Papel y cartón & 5 & 2 & 906 & 1.940 & 301 & 893 & $3,88 \%$ & $5,59 \%$ \\
\hline Productos de la refinación del petróleo & 4 & 2 & 2.175 & 8.820 & 985 & 4.392 & $7,25 \%$ & $7,76 \%$ \\
\hline Productos químicos y farmacéuticos & 18 & 5 & 6.255 & 8.412 & 3.780 & 4.065 & $1,49 \%$ & $0,36 \%$ \\
\hline $\begin{array}{l}\text { Productos minerales no metálicos y } \\
\text { metalúrgicos }\end{array}$ & 6 & 3 & 4.110 & 8.391 & 2.697 & 3.825 & $3,63 \%$ & $1,76 \%$ \\
\hline Aparatos eléctricos y vehículos & 6 & 2 & 3.471 & 4.035 & 2.194 & 1.737 & $0,76 \%$ & $-1,16 \%$ \\
\hline Totales & 81 & 28 & 39.774 & 64.200 & 20.866 & 32.022 & $2,42 \%$ & $2,16 \%$ \\
\hline \multicolumn{2}{|c|}{$\%$ renglones escogidos/renglones seleccionados } & $34,6 \%$ & & & $52,5 \%$ & $49,9 \%$ & & \\
\hline
\end{tabular}

TAG = Tasa anual geométrica para el periodo indicado

a/ Tabla preparada por el estudiante Omar Julián Puerto, para su trabajo de grado en el Semillero de Investigación del Observatorio sobre Desarrollo Humano de la Universidad Autónoma de Colombia. La fuente primaria de la información es la Encuesta Anual Manufacturera del DANE

Al avanzar en la comprobación de la hipótesis para Colombia, en la tabla 4 se presenta el valor de la producción bruta tanto de los 81 renglones seleccionados como de los 28 escogidos, indicando la rama industrial a la que pertenecen. Como se desprende de la tabla 3, la producción bruta de los 81 renglones seleccionados representa cerca de la mitad de la producción total de la industria para los años inicial y final del lapso 1994-2014, pues en ambos equivale a un poco más del $45 \%$. A su vez, los 28 renglones escogidos representan cerca de la cuarta parte de la producción industrial, ya que en el primer año se acerca al $24 \%$ y en el segundo al $23 \%$ de la producción bruta. Puede decirse que el "universo» seleccionado - del cual forman parte los renglones escogidos-es significativo respecto a la producción bruta industrial colombiana.

También la tabla 4 comprueba que el valor de la producción de los renglones seleccionados aumentó al mismo ritmo que la producción industrial total, esto es, al 2,42\% anual, al pasar de 39,8 billones en 1994 a 64,2 billones en 2014, en pesos de 2005; es decir, el valor bruto de la producción de estos 81 renglones, que equivalen a casi la mitad del total de la producción industrial nacional, aumentó en términos monetarios 
reales en casi dos terceras partes, $61,4 \%$, durante el lapso estudiado. Ninguno de los grupos por rama industrial en que se presentan estos 81 renglones muestra disminución, pero si ritmos de crecimiento muy diferentes, que van del $7,25 \%$ anual en los cuatro renglones de productos de la refinación del petróleo, el $3,88 \%$ en los cinco de papel y cartón y $3,63 \%$ en los seis productos de minerales no metálicos y metalúrgicos, hasta $0,76 \%$ en los seis renglones de aparatos eléctricos y vehículos. En el caso de los 28 renglones escogidos, de los 81 seleccionados, la situación es similar, con un incremento global inferior a los seleccionados: muestran una TAG de 2,16\%, con cifra negativa, esto es, disminución absoluta del valor de la producción, en los renglones escogidos de aparatos eléctricos y vehículos ${ }^{6}$.

\subsection{Comportamiento de los renglones escogidos.}

La tabla 5 presenta los 28 renglones escogidos en orden descendente según el incremento en la producción física, durante el lapso 1994-2014 (columna 10 de la tabla). La producción bruta en estos 28 renglones aumentó de $\$ 20,9$ billones en 1994 a $\$ 32,0$ billones en 2014 , un incremento de un poco más de la mitad en los 20 años en valores constantes de 2005 , o sea a una tasa anual acumulativa del $2,16 \%$, inferior a la tasa de los 81 renglones seleccionados, $2,42 \%$ y al incremento de toda la industria, $2,42 \%$. (Véase la tabla 4). Como puede observarse, de los 28 renglones, en 19 la producción en unidades físicas (valores de uso) aumentó a una TAG anual superior al incremento de la producción bruta industrial, que como se muestra en la tabla 3 fue del 2,42\% para ese lapso. Son sorprendentes los incrementos en los cinco primeros renglones, pues van de más de cinco veces en azulejos y baldosas de porcelana a nueve veces en pañales desechables. En estos cinco renglones la disminución en los costos unitarios, medidos en valores constantes de 2005, osciló entre más de $10 \%$ en papel sanitario y $74 \%$ en pañales desechables. En los 15 renglones siguientes los costos unitarios también disminuyeron en guarismos de dos dígitos, en una escala que va de más del $90 \%$

6 La denominación específica de los 28 renglones escogidos puede verse en la tabla 5 . Hay que aclarar que estos cálculos, y todos los que tengan que ver con renglones, se han hecho con base en la información encontrada en la Encuesta Anual Manufacturera del DANE, que a su vez es suministrada por las empresas que responden a la encuesta. Por tanto, la exactitud de estos resultados está sujeta a la veracidad de la información suministrada, aunque se supone que es fidedigna, pues está amparada por la confidencialidad que garantiza el DANE. 
en alimentos balanceados para ganado vacuno hasta casi el $11 \%$ en leche líquida entera. En productos farmacéuticos no es posible este cálculo por la heterogeneidad en la producción; sólo en cinco renglones aumentaron los costos unitarios, medidos en valores constantes. Como es observable, lo anterior es indicativo de los cambios en el consumo de los hogares.

En resumen, en 23 renglones disminuyeron los costos unitarios. La pregunta ineludible es: ¿Esta disminución, que cubre más del $80 \%$ de los renglones escogidos (excluidos productos farmacéuticos), si bien ocurrió sobre los costos en producción, esto es, sobre productos puestos en fábrica, se reflejó en los precios finales que pagaron los consumidores? Todo indica que no, si se ve la evolución de los precios en la «canasta familiar» que mide el DANE mes a mes para calcular el índice de precios al consumidor -IPC-. Para tener más seguridad en la respuesta, será necesaria una investigación al respecto. 


\section{Tabla 5}

Renglones escogidos de la producción total de la industria manufacturera 1994 y 2014 en orden de la TAG en la cantidad a/

\section{Producción en cantidad, valor total y costo unitario (valores constantes de 2005)}

\begin{tabular}{|c|c|c|c|c|c|c|c|c|c|c|c|c|}
\hline \multirow{3}{*}{$\begin{array}{c}\text { ARTíCULOS } \\
\text { (con producción superior a } 200 \text { mil } \\
\text { millones de } \$ \text { de } 2005 \text { en 2014) }\end{array}$} & \multirow{3}{*}{$\begin{array}{l}\text { Unidad } \\
\text { de } \\
\text { medida }\end{array}$} & \multicolumn{2}{|c|}{ PRODUCCIÓN 1994} & \multicolumn{4}{|c|}{ PRODUCCIÓN 2014} & \multirow{3}{*}{\begin{tabular}{|c|} 
Reduc- \\
ción en \% \\
del costo \\
unitario \\
de 1994 a \\
2014
\end{tabular}} & \multirow{3}{*}{$\begin{array}{c}\text { TAG } \\
\text { Cantida- } \\
\text { des } 1995 \\
2014\end{array}$} & \multirow{3}{*}{\begin{tabular}{|c|} 
TAG \\
Valores \\
$1995-$ \\
2014
\end{tabular}} & \multirow{3}{*}{\begin{tabular}{|c} 
Incremen- \\
to $\%$ \\
$2014 / 1994$ \\
en cantida- \\
des
\end{tabular}} & \multirow{3}{*}{$\begin{array}{l}\text { Incremen- } \\
\text { to } \% \\
2014 / 1994 \\
\text { en valores }\end{array}$} \\
\hline & & \multirow{2}{*}{$\begin{array}{c}\text { CANTIDAD } \\
\text { Miles, } \\
\text { unidad de } \\
\text { medida } \\
\text { asignada }\end{array}$} & \multirow{2}{*}{\begin{tabular}{|c|} 
VALOR \\
TOTAL \\
Miles de \\
millones \\
de $\$ 2005$ \\
\end{tabular}} & \multicolumn{2}{|c|}{ CANTIDAD } & \multicolumn{2}{|c|}{ VALOR TOTAL } & & & & & \\
\hline & & & & $\begin{array}{c}\text { Miles, } \\
\text { unidad de } \\
\text { medida } \\
\text { asignada }\end{array}$ & $\begin{array}{c}\text { INDICE } \\
\text { Base }=100 \\
\text { año } 1994\end{array}$ & $\mid \begin{array}{l}\text { Miles de } \\
\text { millones } \\
\text { de } \$ 2005\end{array}$ & $\begin{array}{c}\text { INDICE } \\
\text { Base }=100 \\
\text { año } 1994\end{array}$ & & & & & \\
\hline Pañales desechables & $\mathrm{mi}$ & 273 & 226 & 2.576 & 943,6 & 553 & 244,7 & 74,1 & $11,88 \%$ & $4,58 \%$ & $843,6 \%$ & $144,7 \%$ \\
\hline Papel sanitario fraccionado o no & $\mathrm{mi}$ & 146 & 8 & 1.243 & 851,4 & 570 & $6.764,8$ & 10,3 & \begin{tabular}{l|l}
3 & $11,30 \%$ \\
\end{tabular} & $23,46 \%$ & $751,4 \%$ & $6664,8 \%$ \\
\hline Carne de pollo y gallina & $\mathrm{kg}$ & 142.883 & 801 & 950.653 & 665,3 & 2.762 & 344,8 & 48,2 & $9,94 \%$ & $6,38 \%$ & $565,3 \%$ & $244,8 \%$ \\
\hline Toallas sanitarias & $\mathrm{mi}$ & 921 & 186 & 5.661 & 614,7 & 321 & 172,6 & 71,9 & $9,50 \%$ & $2,77 \%$ & $514,7 \%$ & $72,6 \%$ \\
\hline Azulejos y baldosas de porcelana & m2 & 12.722 & 244 & 64.693 & 508,5 & 500 & 205,3 & 59,6 & $8,47 \%$ & $3,66 \%$ & $408,5 \%$ & $105,3 \%$ \\
\hline Azúcar refinada & $\mathrm{kg}$ & 305.340 & 442 & 1.037 .741 & 339,9 & 866 & 195,9 & 42,4 & $6,31 \%$ & $3,42 \%$ & $239,9 \%$ & $95,9 \%$ \\
\hline Jabones de tocador & $\mathrm{kg}$ & 24.410 & 248 & 81.430 & 333,6 & 317 & 127,4 & 61,8 & $6,21 \%$ & $1,22 \%$ & $233,6 \%$ & $27,4 \%$ \\
\hline Pinturas para agua, p.v.a.y similares & $g$ & 9.219 & 192 & 29.926 & 324,6 & 303 & 157,8 & 51,4 & $6,06 \%$ & $2,31 \%$ & $224,6 \%$ & $57,8 \%$ \\
\hline Yogurt & 1 & 69.726 & 359 & 211.791 & 303,7 & 597 & 166,3 & 45,3 & $5,71 \%$ & $2,58 \%$ & $203,7 \%$ & $66,3 \%$ \\
\hline Tuberías de polivinilo & $\mathrm{kg}$ & 43.186 & 405 & 123.148 & 285,2 & 395 & 97,5 & 65,8 & $5,38 \%$ & $-0,13 \%$ & $185,2 \%$ & $-2,5 \%$ \\
\hline Neveras para uso doméstico & $n$ & 465 & 392 & 1.149 & 247,3 & 422 & 107,7 & 56,5 & $4,63 \%$ & $0,37 \%$ & $147,3 \%$ & $7,7 \%$ \\
\hline $\begin{array}{l}\text { Alimentos balanceados para ganado } \\
\text { vacuno }\end{array}$ & $\mathrm{kg}$ & 267.990 & 224 & 627.281 & 234,1 & 353 & 157,6 & 92,5 & $4,34 \%$ & $2,30 \%$ & $134,1 \%$ & $57,6 \%$ \\
\hline Detergentes en polvo & $\mathrm{kg}$ & 92.952 & 502 & 208.210 & 224,0 & 477 & 95,0 & 57,6 & $4,11 \%$ & $-0,26 \%$ & $124,0 \%$ & $-5,0 \%$ \\
\hline Papel Kraft & $\mathrm{kg}$ & 156.910 & 293 & 310.114 & 197,6 & 323 & 110,2 & 44,2 & $3,47 \%$ & $0,49 \%$ & $97,6 \%$ & $10,2 \%$ \\
\hline Alimentos balanceados para aves & $\mathrm{kg}$ & 1.352 .994 & 1.453 & 2.655 .147 & 196,2 & 1.762 & 121,3 & 38,3 & $3,43 \%$ & $0,97 \%$ & $96,2 \%$ & $21,3 \%$ \\
\hline Leche líquida entera & 1 & 985.945 & 1.222 & 1.765 .476 & 179,1 & 1.951 & 159,7 & 10,9 & $2,96 \%$ & $2,37 \%$ & $79,1 \%$ & $59,7 \%$ \\
\hline Carne vacuna, fresca & $\mathrm{kg}$ & 32.246 & 188 & 56.632 & 175,6 & 272 & 144,7 & 17,8 & $2,86 \%$ & $1,86 \%$ & $75,6 \%$ & $44,7 \%$ \\
\hline Automóviles & $\mathrm{n}$ & 51 & 1.802 & 88 & 173,7 & 1.315 & 73,0 & 58,0 & $2,80 \%$ & $-1,56 \%$ & $73,7 \%$ & $-27,0 \%$ \\
\hline Camisetas de tejido de algodón & $\mathrm{n}$ & 20.005 & 212 & 34.347 & 171,7 & 314 & 148,1 & 13,7 & $2,74 \%$ & $1,98 \%$ & $71,7 \%$ & $48,1 \%$ \\
\hline Aceite mezclado para la mesa y la cocina & $\mathrm{kg}$ & 161.260 & 636 & 241.519 & 149,8 & 480 & 75,5 & 49,7 & $2,04 \%$ & $-1,40 \%$ & $49,8 \%$ & $-24,5 \%$ \\
\hline $\begin{array}{l}\text { Bebidas gaseosas no alcohólicas } \\
\text { (maltas, gaseosas, etc) }\end{array}$ & 1 & 2.227 .662 & 2.365 & 3.182 .344 & 142,9 & 3.260 & 137,8 & 3,5 & $1,80 \%$ & $1,62 \%$ & $42,9 \%$ & $37,8 \%$ \\
\hline Cemento gris & $t$ & 8.394 & 1908 & 11.686 & 139,2 & 2.742 & 143,8 & $-3,3$ & $1,67 \%$ & $1,83 \%$ & $39,2 \%$ & $43,8 \%$ \\
\hline Cerveza tipo pilsen & 1 & 1.509 .779 & 2.076 & 2.016 .423 & 133,6 & 3.188 & 153,6 & $-15,0$ & $1,46 \%$ & $2,17 \%$ & $33,6 \%$ & $53,6 \%$ \\
\hline Aceites lubricantes f.d.r. & g & 39.454 & 453 & 52.353 & 132,7 & 741 & 163,8 & $-23,4$ & $1,42 \%$ & $2,50 \%$ & $32,7 \%$ & $63,8 \%$ \\
\hline $\begin{array}{l}\text { Barras y varillas de hierro o acero de } \\
\text { sección circular laminadas en caliente }\end{array}$ & $\mathrm{kg}$ & 427.926 & 546 & 546.757 & 127,8 & 582 & 106,6 & 16,6 & $1,23 \%$ & $0,32 \%$ & $27,8 \%$ & $6,6 \%$ \\
\hline Gasolina motor - corriente & $g$ & 1.085 .350 & 533 & 1.112 .246 & 102,5 & 3.651 & 685,5 & $-568,9$ & $0,12 \%$ & $10,10 \%$ & $2,5 \%$ & $585,5 \%$ \\
\hline Aguardiente & 1 & 107.368 & 518 & 57.260 & 53,3 & 431 & 83,2 & $-56,0$ & $-3,09 \%$ & $-0,92 \%$ & $-46,7 \%$ & $-16,8 \%$ \\
\hline $\begin{array}{l}\text { Productos farmacéuticos, para uso } \\
\text { humano }\end{array}$ & $\mathrm{v}$ & - & 2433 & - & - & 2.574 & 105,8 & & $\mathrm{NC}$ & $0,28 \%$ & $\mathrm{NC}$ & $5,8 \%$ \\
\hline $\begin{array}{l}\text { TOTAL VALORES } 28 \text { renglones } \\
\text { escocidos }\end{array}$ & & & 20.866 & & & 32.022 & & & & $2,16 \%$ & & $53,5 \%$ \\
\hline
\end{tabular}

TAG = Tasa anual geométrica para el periodo indicado

a/ Tabla preparada por el estudiante Omar Julián Puerto, para su trabajo de grado, en el Semillero de Investigación del Observatorio sobre Desarrollo Humano de la Universidad Autónoma de Colombia. La fuente primaria de la información es la Encuesta Anual Manufacturera del DANE 
Pero además habría que tener en cuenta que en la mayoría de estos renglones una o pocas empresas controlan el respectivo mercado, bajo condiciones de monopolio, pues donde hay varias se ha demostrado que existe una competencia simulada, con posición dominante desde principios o mediados del siglo 20; también en algunos de estos renglones se ha comprobado o está en proceso de investigación la cartelización de empresas, esto es, los acuerdos subrepticios para fijar precios o repartirse mercados. Como ejemplos se puede decir que en el caso del aceite para mesa y cocina se encuentra el conglomerado Alianza Team; en pañales, toallas sanitarias y papel el control lo ejercen empresas como Tecnoquímicas, Familia, Kimberly Clark y el conglomerado Carvajal, con más de un siglo de existencia; en azúcar se encuentra el cartel de los ingenios, propiedad de cuatro o cinco familias vallecaucanas, agrupados en Asocaña, que en los últimos años ha estado bajo investigación de la Superintendencia de Industria y Comercio -SIC-; en pinturas y tubería de polivinilo el control lo ejerce el grupo Orbis (antes Mundial); en los renglones de bebidas gaseosas y cervezas el control es casi absoluto por parte del conglomerado Postobón-Lux (del grupo financiero de Ardila Lulle), en el primero, y de Bavaria (propiedad hoy de la empresa mundial $A B I n B e v$, en donde el grupo financiero Santodomingo tiene una participación decisoria), en el segundo; en cemento, el control lo ejerce el cartel compuesto por los conglomerados Argos (del grupo financiero Suramericana), Cemex y Holcim, también bajo investigación de la SIC. Y así podrían encontrarse otros renglones, bajo condiciones similares ${ }^{7}$. En este caso podría aplicarse lo que el Informe Oxfam de 2018 dice respecto a la desigualdad: "(...) cada vez hay más pruebas que demuestran que los niveles actuales de desigualdad no son el resultado del esfuerzo y la toma de riesgos, sino de beneficios inesperados que no se corresponden con la actividad productiva, lo que los economistas denominan 'rentas'". $\mathrm{Y}$ como origen de esos beneficios menciona tres fenómenos, que también han sido determinantes en Colombia: monopolios, clientelismo y herencias (OXFAM, 2018, pág. 40).

Como es natural, esta disminución en los costos unitarios afecta el valor total de la producción del respectivo renglón, haciendo que su

7 Mayor información sobre estos conglomerados y grupos financieros puede verse (Silva-Colmenares, 2004) y (Silva-Colmenares \& Padilla, 2015) 
incremento en todo el lapso sea muy inferior al incremento en unidades físicas, como es evidente al comparar el incremento en el valor, medido en TAG para todo el lapso (columna 11 de la tabla 5), con la tasa que muestra la evolución de la cantidad física (columna 10 de la tabla 5): en 22 de los 27 renglones, excluidos productos farmacéuticos, la producción en unidades físicas crece a mayor ritmo que la producción en valores constantes. Es decir, mientras aumenta la producción industrial en valores de uso, disminuye, ya sea en forma absoluta o relativa, la producción en valores de cambio, entendida para este ejercicio como la producción industrial valorizada en unidades monetarias constantes. Aunque puede ser necesario hacer análisis de mayor cobertura, el ejercicio realizado hasta este punto permite afirmar que se comprueba, de alguna manera, la hipótesis planteada en un párrafo anterior.

\subsection{Pierde peso relativo el trabajo humano «vivo» en el valor agregado industrial.}

Pasando a otro aspecto, si bien no puede decirse con seguridad que los consumidores no se han beneficiado en forma proporcional de la disminución en los costos --como resultado de la elevación de la productividad bruta--, en cambio sí existe la posibilidad de mostrar que tampoco fueron los trabajadores de la industria -tomada como ejemplo-- los beneficiados, pues en un artículo publicado en la revista Apuntes del CENES (Silva-Colmenares \& Padilla, 2017) ${ }^{8}$ comprueban que en la composición del valor agregado industrial la participación de los trabajadores ha venido disminuyendo en las últimas décadas, según ejercicio sobre el particular. Si al total del valor agregado se resta el total de la remuneración a los trabajadores, o sea el «trabajo vivo», la porción restante del valor agregado corresponde a las ganancias empresariales y los ingresos de otros factores no salariales ni empresariales, en sí; los autores del artículo denominan a esta porción, que es el resto del valor agregado, Excedente Bruto. Aunque el artículo mencionado muestra la información desde el año 1975, y el acumulado por gobiernos, para los fines de esta nota sólo se toma el lapso comprendido desde el gobierno de Ernesto Samper (1995-1998) hasta la primera administración de Juan

8 Silva-Colmenares Julio y Padilla Pardo Carolina, La industria colombiana 1975-2014: inequidad entre trabajo y capital, revista Apuntes del CENES, UPTC Tunja, vol. 36 No. 63 enero-junio 2017, pp. 137-167. 
Manuel Santos (2011-2014), para que coincida con el periodo utilizado en las tablas anteriores. (Los porcentajes de la última línea de la tabla 6 corresponden al cambio ocurrido entre el acumulado en el gobierno de Santos y el acumulado en el gobierno de Gaviria Trujillo, el gobierno anterior al lapso estudiado).

\section{Tabla 6}

Industria 1995-2014 -Composición del valor agregado e indicadores seleccionados por trabajador ocupado

Acumulado en el cuatrienio de cada gobierno, en valores de constantes de 2005

\begin{tabular}{|l|r|r|r|r|r|r|}
\hline \multicolumn{1}{|c|}{ GOBIERNOS } & VA bn\$ & \% RT/VA & \% EB/VA & $\begin{array}{c}\text { RT pc } \\
\text { mn\$ }\end{array}$ & $\begin{array}{c}\text { VA pc } \\
\text { mn\$ }\end{array}$ & $\begin{array}{c}\text { EB pc } \\
\text { mn\$ }\end{array}$ \\
\hline Ernesto Samper Pizano 1995-1998 & 168,7 & $29,3 \%$ & $70,7 \%$ & 94,8 & 269,8 & 190,8 \\
\hline Andrés Pas trana Arango 1999-2002 & 157,1 & $25,4 \%$ & $74,6 \%$ & 89,9 & 295,3 & 220,3 \\
\hline Álvaro Uribe Vélez 2003-2006 & 187,7 & $19,5 \%$ & $80,5 \%$ & 84,6 & 324,1 & 260,9 \\
\hline Álvaro Uribe Vélez 2007-2010 & 214,9 & $19,1 \%$ & $80,9 \%$ & 84,7 & 330,9 & 267,8 \\
\hline Juan Manuel Santos 2011-2014 & 219,6 & $19,8 \%$ & $80,2 \%$ & 80,7 & 323,4 & 259,3 \\
\hline$\%$ variación 2011-2014/1991-1994 & $39,9 \%$ & & & $-9,8 \%$ & $25,7 \%$ & $44,7 \%$ \\
\hline
\end{tabular}

Fuente: Silva-Colmenares Julio y Padilla Pardo Carolina, La industria colombiana 1975-2014: Inequidad entre trabajo y capital, revista Apuntes del CENES, Vol. 36, No. 63 enero-junio 2017, datos parciales tomados de las tablas 1 (p.142) y 2 (p.145).

$\mathrm{VA}=$ Valor agregado

RTNA = Remuneración total a trabajadores sobre valor agregado

$E B / N A=$ Excedente bruto sobre valor agregado (VA-RT)

$\mathrm{RT} p \mathrm{pc}=$ Remuneración promedio anual en el cuatrienio por trabajador ocupado remunerado

$\mathrm{VApc}=$ Valor agregado promedio anual en el cuatrienio por trabajador ocupado

$E B$ pc $=$ Excedente bruto promedio anual en el cuatrienio por trabajador ocupado bn $\$=$ Billones de pesos de 2005

$\mathrm{mn} \$=$ Millones de pesos de 2005

Como muestra la tabla 6, durante los gobiernos que cubren el periodo 1995-2014 (20 años, cinco gobiernos) el valor agregado, en pesos constantes de 2005, aumentó de manera continua, excepto en el gobierno de Pastrana Arango --durante el cual Colombia vivió una fuerte depresión económica--, siendo el promedio anual alcanzado en la primera administración de Santos un $40 \%$ superior a la cifra que se logró como promedio en la administración de Gaviria. En confirmación de este aumento, puede recordarse que según la tabla 1 el PIB de la industria pasó de \$36,4 billones en 1994 a $\$ 57,2$ billones en 2014, en valores constantes de 2005, lo que representó un incremento real del $57 \%$ en los 20 años. Pero mientras aumentaba el valor agregado en la 
industria, la distribución entre los trabajadores y las distintas modalidades de empresarios y otros factores que participan del valor agregado en la industria, que en categorías de la Economía Política se denomina la distribución entre trabajo y capital, tendía a una creciente desigualdad; si al inicio del periodo la distribución era, en términos generales, de 30-70, al final llegó a 20-80, como se desprende de las columnas 3 y 4 de la tabla 6 . Es decir, las ganancias empresariales y de otros factores equivalen a cuatro veces la remuneración a los trabajadores, lo que a todas luces es una situación inequitativa, existente en muy pocos países del mundo, aunque no es éste el momento de comprobar esta afirmación.

Como dice el informe de Oxfam de 2018, "Para poner fin a la actual crisis de desigualdad, es necesario que todos los trabajadores y trabajadoras del mundo disfruten de salarios y trabajos dignos. En todo el mundo, la economía del $1 \%$ más rico se construye a expensas de trabajos mal pagados, a menudo ocupados por mujeres, que reciben salarios miserables sin que se respeten sus derechos fundamentales. (...)" (OXFAM, 2018, pág. 9). En sentido similar se expresa el filósofo estadounidense Michael Sandel (1953- ) quien en entrevista para el diario El Tiempo (Bogotá), ante la pregunta ¿Cuál es la relación entre la corrupción, la inequidad y los términos del debate público?, respondió: "(...) Estas son fundamentalmente preguntas no solo de la ley, sino valores y normas y actitudes morales, y por eso la corrupción y la inequidad son problemas interrelacionados, ambos hacen que la confianza cívica se pierda, que sea difícil, y para entenderla es necesario tener un debate acerca de la ética pública”. Y más adelante, a la pregunta ¿Cuáles son los elementos que debe tener el debate democrático?, respondió: "Mi sugerencia es que un debate público rico debe responder unas preguntas que le importan a la gente, por ejemplo: ¿Qué es lo que hace una sociedad justa? ¿Qué es lo que hace la inequidad? ¿Qué es lo que nosotros debemos hacer como ciudadanos?" (EI Tiempo, 2018).

Pero es tal el poder de la oligarquía financiera, que ni siquiera en los países de América Latina en donde ha habido gobiernos llamados, en términos generales, de izquierda, en lo que se ha denominado la «marea rosa», ha habido cambios sustanciales. Como reconoce un artículo al respecto, "Está comprobado que dichos gobiernos fueron capaces 
de reducir de manera significativa la pobreza, pero no disminuir la concentración del ingreso y de riqueza en el pequeño grupo de millonarios situado en la cúspide de la pirámide social de cada país". Y después de señalar algunas razones explicativas, concluye diciendo que "Son, todas ellas, explicaciones sólidas y pertinentes que merecen ser tomadas en consideración. Sin embargo, revelan tan solo la superficie del fenómeno y no dilucidan las razones últimas por las que los gobiernos de izquierda no han ido mucho más allá de distribuir efectivo a los pobres. Para comprender estas razones profundas, es necesario articular el análisis de las desigualdades sociales con el examen, en cada caso, de las relaciones de poder". Sólo así podrá entenderse "por qué la cúspide de la pirámide social (el 1\% más rico de cada país) ha sido capaz de ampliar su participación en la apropiación de la riqueza y los ingresos (...)" (Badia i Dalmases \& Costa, 2018). Como bien lo dijo el juez Louis Brandeis, de la Corte Suprema de los Estados Unidos, y lo recuerda el informe Oxfam para el Foro Económico Mundial de 2018, "en este país, podremos tener democracia o podremos tener una enorme riqueza concentrada en las manos de unos pocos, pero no podremos tener ambas cosas a la vez'. Nuestros líderes son conscientes de ello, pero en lugar de adoptar medidas para reducir la concentración de la riqueza y la desigualdad, están más interesados en restringir la democracia y la libertad de expresión para demandar una sociedad más justa" (OXFAM, 2018, pág. 11).

Viendo la distribución del valor agregado que muestra la tabla 6 bajo otras consideraciones, las tres últimas columnas confirman que lo ocurrido en la industria colombiana en estos 20 años no fue tanto un proceso de desindustrialización -sin negar su disminución en el peso relativo respecto al PIB y la PO--, sino de cambio en el uso del trabajo humano y otros insumos en el proceso de producción, que llevaron a un evidente aumento en la productividad bruta, lo que explica la disminución en los costos unitarios de producción en un conjunto de renglones en donde es posible calcular este indicador, y que representan cerca del $25 \%$ de la producción industrial, como también se analizó en párrafos anteriores. Un par de cifras son contundentes al respecto. Mientras el valor agregado generado por trabajador ocupado, como promedio anual, aumentó en casi $26 \%$ (columna 6 de la tabla 6 ) y el excedente bruto de explotación en cerca de $45 \%$ (columna 7 de la tabla 6 ), en el 
lapso transcurrido entre la administración de Gaviria y la administración de Santos, la remuneración anual promedio por trabajador disminuyó en casi $10 \%$. Una situación similar se observa a nivel mundial; como dice el informe (OXFAM, 2018, pág. 16) "(...) Si bien el valor de lo que producen los trabajadores y trabajadoras ha aumentado significativamente, esto no se ha traducido en mejoras salariales o condiciones laborales. La OIT analizó 133 países ricos y pobres, durante el periodo comprendido entre 1995 y 2014, y encontró que, en 91 países, el aumento de la productividad y el crecimiento económico no se vieron acompañados de un aumento de los salarios".

\section{CONCLUSIONES}

En las páginas anteriores se ha comprobado que, si bien el PIB Producto Interno Bruto- y la PO - Población Ocupada - industrial han disminuido como proporción del total nacional, no es porque hayan disminuido en valores absolutos tales variables, sino porque otros sectores de la economía han crecido a mayor ritmo. La tabla 3 muestra que la producción total de la industria aumentó al $2,42 \%$ en tasa anual acumulativa durante el lapso 1995-2014 (20 años), al tiempo que 81 renglones seleccionados, que representan cerca de la mitad de la producción industrial, crecieron a un promedio del $2,42 \%$ en TAG y 28 renglones escogidos, de esos 81 , que equivalen a casi la cuarta parte de la producción industrial, lo hicieron al $2,16 \%$ en TAG, como lo muestra la tabla 4. Enfocado el análisis en los 28 renglones escogidos, en razón a que la unidad de medida fue homogénea durante todo el lapso, la tabla 5 comprueba que la producción industrial en unidades físicas, esto es, en valores de uso, aumenta a mayor ritmo que la producción en valores de cambio, entendido como el valor en precios constantes (columnas 10 y 11 de la tabla 5), lo que indica una reducción en los costos unitarios de producción, en algunos casos reducciones muy importantes (columna 9 de la tabla 5). Todo indica que por cambios tecnológicos en los procesos de trabajo, o modificaciones en las materias primas o los insumos utilizados en la producción o por cambios en la fuerza de trabajo, entre los principales, ha habido un incremento en la productividad bruta, como señala la hipótesis que se plantea en este trabajo, aunque es posible que sea necesario ampliar este análisis. 
Las tabla 6 comprueba que en esos 20 años los propietarios de los medios de producción y de otro tipo de activos lograron redistribuir en su favor el valor agregado en la industria manufacturera, pues el excedente bruto (valor agregado menos remuneración a los trabajadores) pasó del $70,7 \%$, como promedio en la administración Samper Pizano, al 80,2\%, también como promedio, en el primer gobierno de Santos Calderón (columna 4 de la tabla 6). En consecuencia, mientas aumentó el valor agregado y el excedente bruto generado por cada trabajador, como promedio anual en cada cuatrienio, disminuyó la remuneración recibida por cada trabajador, también como promedio (columnas 5, 6 y 7 de la tabla 6). En otras palabras, sobre los trabajadores industriales se descargó la disminución en el peso relativo del sector en el PIB total, llevando a su empobrecimiento, como clase social. O sea, mientras la industria disminuía su peso relativo en el PIB, las ganancias empresariales industriales aumentaban, tanto en términos relativos como absolutos. Lo que no niega que en otras actividades económicas, que aumentaron su peso relativo en el PIB, la tasa de ganancia empresarial puede ser mayor que en la industria.

Pero el mundo, incluida Colombia, no está condenado a que se agrave este panorama, que cada vez es menos sostenible. Un mundo mejor es posible, pues como dice el párrafo inicial del acápite final del informe (OXFAM, 2018, pág. 64), "Existen alternativas a la estructura actual de la economía. Es posible vencer estas poderosas fuerzas económicas. Podemos crear una economía más humana, que anteponga los intereses de los trabajadores y pequeños productores, y no los de quienes reciben remuneraciones astronómicas, ni de las grandes fortunas. Sería una economía en la que todas las personas podrían vivir dignamente y al mismo tiempo permitiría preservar el futuro de nuestro planeta. Debemos rechazar los dogmas de la economía neoliberal y la inaceptable influencia que ejercen las élites sobre nuestros gobiernos". 


\section{REFERENCIAS}

(1) Badia i Dalmases, F., \& Costa, S. (enero de 2018). Desigualdad persistente: el controvertido legado de la "marea rosa» en América Latina. Obtenido de Revista Nueva Sociedad: http://nuso.org/articulo/desigualdad-persistente-elcontrovertido-legado-de-la-marea-rosa-en-america-latina/

(2) Clavijo, S., Vera, A., \& Fandiño, A. (2012). La desindustrialización en Colombia. Análisis cuantitativo de sus determinante. Bogotá: Asociación Nacional de Instituciones Financieras -ANIF-.

(3) El Tiempo. (16 de febrero de 2018). El debate político hoy no inspira a nadie. El Tiempo, pág. 2.8.

(4) Marx, C. (1959). El Capital. Crítica de la Economía Política. 2da edición en español. México: Fondo de Cultura Económica.

(5) OXFAM. (2018). Premiar el trabajo, no la riqueza. Para poner fin a la crisis de desigualdad, debemos construir una economía para los trabajadores, no para los ricos y poderosos. . . Davos: OXFAM.

(6) Puerto, O. J. (2017-2018 de En proceso de elaboración). Industria Colombiana 1994-2014: Comportamiento de la producción en unidades físicas y valores constantes - Renglones seleccionados. Trabajo de grado. Bogotá: Universidad Autónoma de Colombia.

(7) Silva-Colmenares, J. (2004). El gran capital en Colombia. Proyección al siglo $X X I$. Bogotá: Planeta.

(8) Silva-Colmenares, J. (2013). Globalización, crecimiento y desarrollo,. En J. (. Silva-Colmenares, Colombia: Crecimiento económico y desarrollo humano: Algunas características del proceso en el lapso 1959-2010 (págs. 87-166). Bogotá: Academia Colombiana de Ciencias Económicas. 
(9) Silva-Colmenares, J. (2014). Divergencia entre el valor y precio del trabajo. ¿Una explicación a la inequidad en la distribución de la riqueza creada? Criterio Libre, Vol. 12 No. 21 , Julio-Diciembre, 23-61.

(10) Silva-Colmenares, J. (2015). Divergencia entre valor y precio del trabajo. Un complemento a la obra El capital en el siglo XXI de Piketty. En Varios, Piketty y los economistas colombianos. Debate sobre El Capital en el siglo XXI (págs. 379-558). Bogotá: Ediciones Aurora-Academia Colombiana de Ciencias Económicas.

(11) Silva-Colmenares, J., \& Padilla, P. C. (2015). Transnacionalización de empresas colombianas. Conglomerados que cruzan fronteras. Bogotá: Universidad Autónoma de Colombia.

(12) Silva-Colmenares, J., \& Padilla, P. C. (2017). La industria colombiana 19752014: inequidad entre trabajo y capital. Apuntes del CENES, UPTC Tunja, vol. 36 No. 63, enero-junio , 137-167. 